\title{
A spectral clustering method of taking the space topology relationship and the voltage data dependency of the power network into consideration
}

\author{
Mei Dong, Tang Zhaohui, Chen Jingting \\ Modern Energy Systems and Power Electronics, , Yuelu Street, Changsha 410083, China Central \\ South University \\ School of Information Science and Engineering, Central South University, Yuelu Street, Changsha \\ 410083, China \\ meidong@csu.edu.cn, zhtang@mail.csu.edu.cn,956976504@qq.com
}

Keywords: Spectral clustering; Graph theory; Spatiotemporal Association Border Set

\begin{abstract}
The voltage is one of the important criteria for the evaluation of the electric energy quality, as well as the sign of reflecting the reactive power balance and reasonable distribution electric power system. Distributed voltage control is a kind of fast and effective controlling method. To deal with the complicated conditions of the divided boundary, this article leads into the concept of the temporal and spatial boundary constraint set, putting forward the method of spectral clustering partition of the boundary constraint set. Firstly, the way of obtaining the distributed numbers is given specific to the electric power net. Then, the voltage time series analysis is carried on the base of the space relationship. Betweenness, degree and other complex internet and intranet concept have been brought in to evaluate the importance of node, the definition of the partition degree has been made through the data visualization. Dividing the boundary node into whirlpool node and isolated node, dealing with them separately. When the voltage fluctuation takes place, the reason can be made clear, the prediction about chain fluctuation spreading tendency will be made and the regulation and control scheme will be given. Application in some province's electric power net system has saved the computing time and improved the response speed.
\end{abstract}

\section{Introduction}

The voltage is one of the important evaluations of the electric energy quality, as well as the sign of reflecting the reactive power balance and reasonable distribution electric power system. One of the traffic control staff's major tasks is to modify the voltage and make sure it to be stable. In the actual power supply practice, the electric power system will limit the threshold of the voltage in the given period, the fluctuation within plus or minus $5 \%$ is normal in common, but adjustment is needed when the fluctuation is too big. 


\subsection{Partition regulation and control}

Because the scale of the actual electric power net is too large, it will be very time-consuming if regulate and control it via all-net calculation, which means it can not meet the need of the rapidity of the emergent regulation and control. In practical application, the change of control amount has obvious effect on the near-by area voltage, but smaller effect on far-distance area, which reflects its apparent social attribute. Therefore, the partition regulation and control has been an important researching direction.

So far, the research on the internet node is divided into the way based on mechanism modeling and the way based on the graph theory. In the electric power system, the mechanism modeling is mainly the power flow analysis with high complexity, with the expanding of the net, a proper result is hard to be got. As to the graph theory, using K- Means and so on, because these algorithms can not consider the attribute of specific net. In this background, some complicated-net's researchers have started to deal with specific physical-meaningful graph theory segment problems with the spectral clustering.

\subsection{The regulation and control method on voltageartition regulation and control}

There are optimal planning way and sensitivity way of voltage regulation and control. Optimization Programming leads to the controlling scheme through working out the mathematic model, which owns well generality, but the computing time is long, and there may be astringency problems, which limits its appliance in emergent control; the sensitivity arithmetic doesn't need the iterative computation, which means there is no astringency problems, so the minimal amount of the adjustment and the adjust equipment can be achieved easily. But on the problem of choosing the control point and ascertain of the controlling amount, only the node can be the accordance, no other nodes' situation will be taken into the consideration. To avoid the over-limit chain, it should be ensured that no new over-limit will appear in the adjustment, therefore, when choosing the control point and ascertaining the control amount, the constraint effect of the normal nodes in the system should be considered; while the real system scale is huge, if taking all of the nodes in the system into the consideration, the overall network calculation will be needed, but a time-consuming calculation can not meet the need of emergency control. How to find out the volatile nodes in the adjustment, and the research on the strategy ensuring these nodes' being in the limit is greatly meaningful to avoidance of the over-limit chain.

\section{The boundary constraint and the spectral clustering}

\subsection{The definition of the Spatiotemporal Association Border Set}

The traditional graph theory way, such as the K-Means and so on, can not consider the features of specific net. And the electric nodes distribution has features like the distribution areas concentrate, the disproportionation of the offering and the need of the electricity and so on. The specific analysis of the specific net is needed. And the voltage data changes all the time, so the realtime regulation and control is needed. And the relation of partitioned boundary nodes and adjacent section can be close or far, which should be judged by time order. For this reason, this article defines the Spatiotemporal Association Border Set (SABS), aimed to the voltage, analyzing different boundaries to fit the reality of the electric power net better. 


\subsection{The concept of the Spectral Clustering}

The Spectral Clustering (SC) is a clustering way based on the graph theory. It divides the weighted graph into two or more optimal sub-graphs to make the interiors of the sub-graphs be similar to each other to the most extent, and the distance between the sub-graphs shall be far to the most extent to reach the common purpose of clustering. The concept of optimal in it means the optimal objective function, which can be the smallest cut, or the cut with the similar size as well as the smallest cut edges, such as which in picture 2-1, or the best cut or normal cut in picture 2-1.

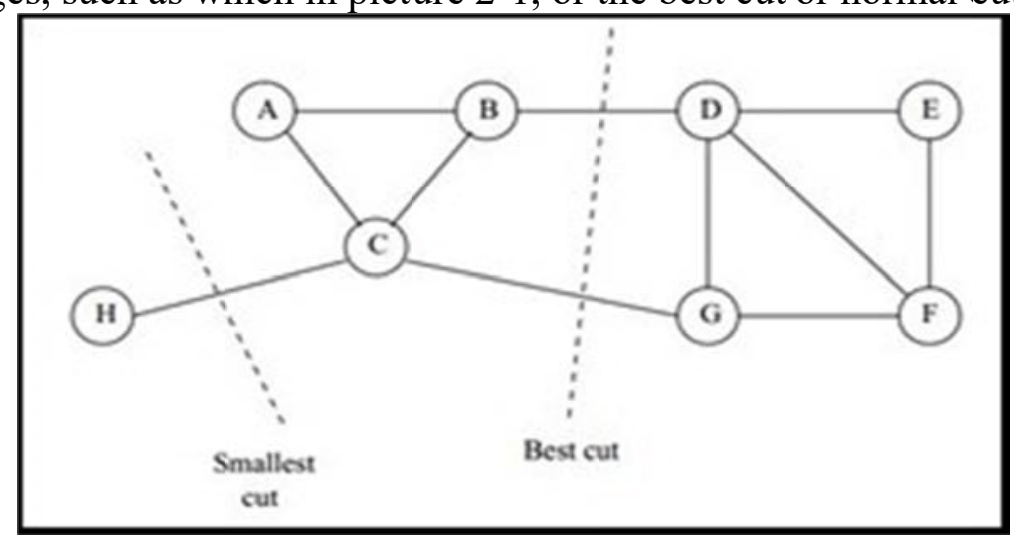

Figure2-1 Spectral clustering schematic figure

\subsection{Algorithm features}

Spectral clustering using graph theory, matrix theory as a supplement to the data, the sample as a vertex, similarity between the samples as weighted edges, thus clustering problem into graph partitioning problem: to find a method of graph partitioning makes the weight of the edges connecting different groups as low as possible, the edge weight group as high as possible ${ }^{[11]}$. And this is very similar to the grid partition ${ }^{[12]}$. The core formula is as follows: $2-1$ :

$$
\operatorname{cut}\left(A_{1}, \ldots, A_{k}\right):=\frac{1}{2} \sum_{i=1}^{k} w\left(A_{i}, \bar{A}_{i}\right)
$$

Which represents a point other than the map (called the complement).

Represents the sum of the weights of all edges of a join and a point.

The $1 / 2$ is introduced in order to reduce the repeated calculation.

\section{Establish the spectrum clustering matrix}

In order to partition the grid, it is necessary to establish the spectral clustering matrix.

\subsection{Power grid topology and transformation}

The study object of this article is a grid voltage data of 500KV site. Contains $17500 \mathrm{KV}$ power station, 7 power supply stations and 4 provincial power supply lines. Data sampling five minutes at a time.

Transformation rule:1)the existing $17500 \mathrm{KV}$ power station site into electrical nodes.2)the existing 7 power supply stations and 4 foreign power lines into power supply node, a total of 11 .

Weighted adjacency matrix:

If there is a node, the weighted adjacency matrix is a square matrix with the following properties:

$$
A_{i, j}= \begin{cases}0 \quad i=j \\ w_{i, j} & i \neq j \text { and }\left(v_{i}, v_{j}\right) \in E \\ \infty & i \neq j \text { arfd }\left(v_{i}, v_{j}\right) \in E\end{cases}
$$


Which represents the weight of the edge; there is no branch between the representation and the representation of the set of edges.

\subsection{Power grid topology and transformation}

The sparse matrix is constructed by using each edge as a matrix, and the sparse matrix is read by matlab. The depth and priority algorithm is used to find the tree and tributary structures, and the adjacency matrix is constructed.

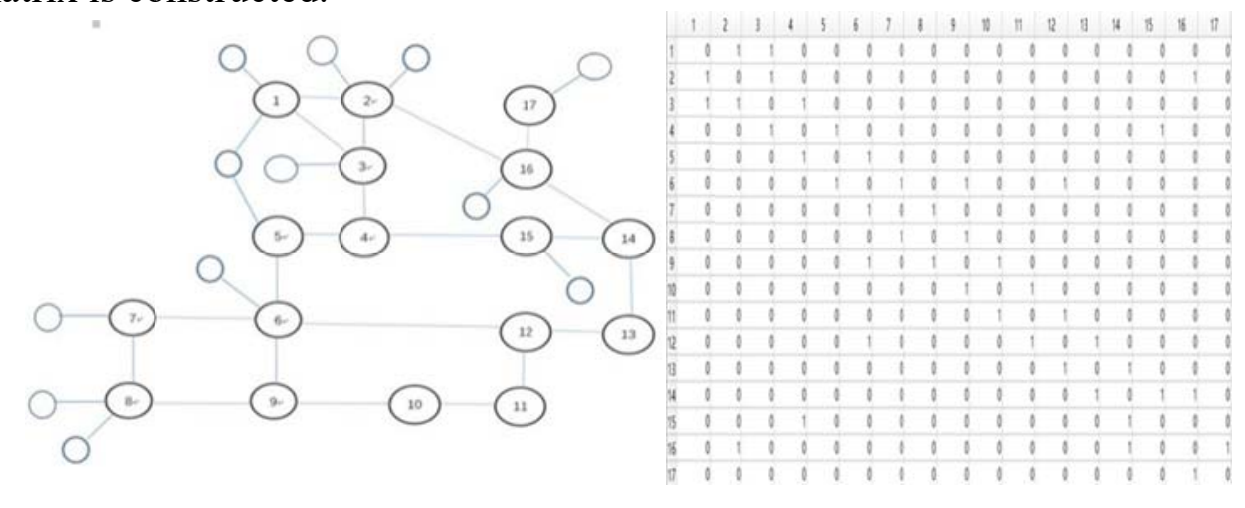

Figure 3-1 Topology model of Tutu figure Figure 3-2 Connection matrix figure

Step:

1) Constructs a $N \times N$ weight matrix $W, w_{i J}$ represents the similarity between sample $i$ and sample $j$, and it is clear that $W$ is a symmetric matrix.

2) Construct a diagonal matrix $D, d_{i j}$ the sum of the elements of the Dth $i$ column. Finally construct the Laplace matrix: $L=D-W$. It can be shown that $L$ is a semi definite and symmetric matrix. Find the eigenvalue of the former $K$ small eigenvalue. Place $K$ feature vectors together to construct an $N \times K$ matrix.The new sample points for K-Means clustering. Where $K$ is divided into $K$ groups, combined with practical significance, the partition is to simplify the power grid regulation, so each partition should be as appropriate as the average. So the following improvements:

$$
\operatorname{RatioCut}\left(A_{1}, \ldots, A_{k}\right):=\frac{1}{2} \sum_{\mathrm{i}=1}^{\mathrm{k}} \frac{\mathrm{w}\left(\mathrm{A}_{i}, \overline{\mathrm{A}_{l}}\right)}{\left|\mathrm{A}_{i}\right|}=\sum_{\mathrm{i}=1}^{\mathrm{k}} \frac{\operatorname{cut}\left(\mathrm{A}_{i}, \overline{\bar{A}_{\mathrm{L}}}\right)}{\left|\mathrm{A}_{i}\right|}
$$

Where $\left|A_{i}\right|$ represents the number of vertices contained in the $A_{i}$ group, and the other symbols are consistent with Equation 2-1.

\section{Realization of spectral clustering partition}

According to the characteristics of the grid, the nodes have the characteristics of highaggregation community network in complex network theory, such as the distribution of nodes in the provincial capital area, and the sparse distribution of the southwest nodes ${ }^{[13]}$. According to the actual situation, the number and density of nodes in different regions provides a reference to the partition. 


\subsection{Node importance indicators}

About how to determine the number of partitions, most existing spectral clustering methods can only estimate the number of one cluster, or estimate the number of non-unique clusters based on the intrinsic gap. In this paper, a non-unique clustering number determination method based on eigenvalue ratio and number distribution is proposed for the grid situation. Meila et al. $(2001,2003)$ have theoretically supported the method ${ }^{[14]}$. Introduction to betweennesse and degree:

The node number is defined as the ratio of the number of paths through the node to the shortest path in all the shortest paths in the network. The node degree refers to the number of edges associated with the node. For a directed graph, the entry of a node is the number of sides entering the node; the degree of the node is the number of edges from that node.

Specific steps are as follows:1)Calculate the percentage of betweenness, find the collection of minimum amount of nodes whose summation is over 50 percent, assume the number as G; 2)Calculate the adjacency matrix, rank the eigenvalues in descending order, analyze the transitions, give the reference partition number, and count it to be number $\mathrm{K}$.

\subsubsection{Calculation steps}

A. To a node h, determine the situation that whether it is located on the shortest path between the point $\mathrm{i}$ and $\mathrm{j}$

1) Assume that the control node is $i$, then node $i$ is treated as the root node of the entire topology network, and convert the network diagram to a tree. $\mathrm{j}$, which is $\mathrm{N}$ nodes far from the node $\mathrm{i}$, is called n-level sub-node (if there is a loop, then take a shorter part between $\mathrm{i}, \mathrm{j}$ as a standard).

2) For each control node, search out the shortest distance of it to other nodes in accordance with the depth search method. And record the points on the shortest distance.

B. The connection conditions of a voltage node in some province are shown in the following table:

Table 4-1 Connection tables between nodes Table

\begin{tabular}{|c|c|c|c|c|c|c|c|c|c|c|c|c|c|c|c|}
\hline Origin node & 1 & 2 & 3 & 4 & 5 & 6 & 7 & 8 & 9 & 10 & 11 & 12 & 13 & 14 & 16 \\
\hline $\begin{array}{c}\text { Connected } \\
\text { node }\end{array}$ & 2,3 & 16 & 4 & 5,15 & 6 & $7,9,12$ & 8 & 9 & 10 & 11 & 12 & 13 & 14 & 15,16 & 17 \\
\hline
\end{tabular}

According to the previous comprehensive transmission of the number of calculation methods can get a 17-node system 21 lines of normalized transmission of the average number of transmission parameters of the order, the situations of nodes located in the shortest path are as follows:

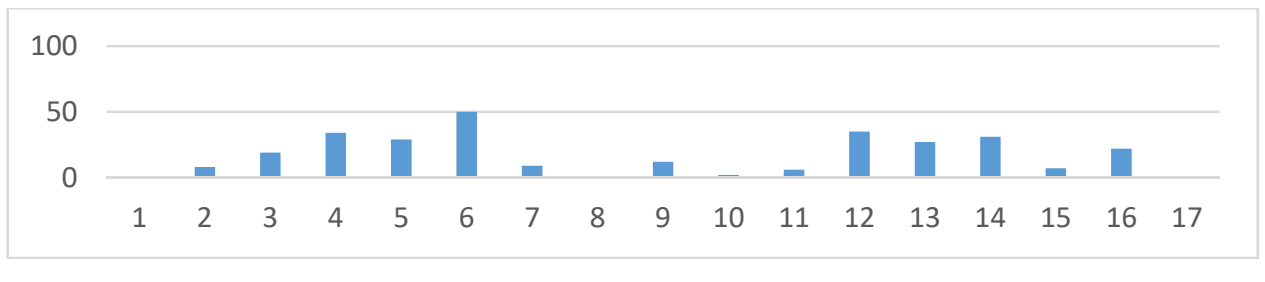

Figure 4-1 Shortest Path Count figure

Figure 3-1 Analysis: No. 1 and No. 2 node only connect to the No.3 node, and constitute a triangle. So when a path passes the No. 1 node, then it must be able to go directly through node 2 and 3 . Node 8 only connects to node 7 and node 9 , which is equivalent to node 6 , No.6 can replace the role of No. 8. As for node 17, it is connected only to node 16. Get the following table: 
Table 4-2 Shortest Path Percentage Table

\begin{tabular}{|c|c|c|c|c|c|c|c|c|c|c|c|c|c|c|c|c|c|}
\hline Node number & 1 & 2 & 3 & 4 & 5 & 6 & 7 & 8 & 9 & 10 & 11 & 12 & 13 & 14 & 15 & 16 & 17 \\
\hline percentage & $0.00 \%$ & $2.75 \%$ & $6.53 \%$ & $11.60 \%$ & $9.97 \%$ & $17.18 \%$ & $3.09 \%$ & $0.00 \%$ & $4.12 \%$ & $0.699 \%$ & $2.06 \%$ & $12.03 \%$ & $9.28 \%$ & $10.65 \%$ & $2.41 \%$ & $7.50 \%$ & $0.00 \%$ \\
\hline Sequence & 15 & 11 & 8 & 3 & 5 & 1 & 10 & 15 & $g$ & 14 & 13 & 2 & 6 & 4 & 12 & 7 & 15 \\
\hline
\end{tabular}

\subsubsection{Eigenvalue analysis}

In Table 4-3, 17 eigenvalues of the Laplace matrix are listed, and the eigenvalue ratio $\beta=$ $\lambda_{i} / \lambda_{j}(i<j<n ; i, j \in N)$ is defined to measure the magnitude of the change between the eigenvalues. The ratio between the eigenvalues are almost all greater than $0.8, \lambda_{5} / \lambda_{6}$ is significantly smaller than the other proportions, thus it can can be considered that there is a transition, so add a partition. Similarly, $\lambda_{8} / \lambda_{9}$ is also the case, add one more partition, the better partition number is expected to be 2 to 4

Table 4-3 Eigenvalue table

\begin{tabular}{|l|l|l|l|l|l|l|l|l|l|l|l|l|l|l|l|l|l|}
\hline Eigenvalue number & 1 & 2 & 3 & 4 & 5 & 6 & 7 & 8 & 9 & 10 & 11 & 12 & 13 & 14 & 15 & 16 & 17 \\
\hline Value & 0 & 0.18 & 0.59 & 0.7 & 0.82 & 1.21 & 1.49 & 2.18 & 2.52 & 2.82 & 3 & 3.17 & 3.93 & 4.26 & 4.43 & 5.06 & 5.64 \\
\hline$\lambda_{2} / \lambda_{i}(i>2)$ & & 1 & 0.3 & 0.25 & 0.22 & 0.15 & 0.12 & 0.08 & 0.07 & 0.06 & 0.06 & 0.05 & 0.05 & 0.04 & 0.04 & 0.04 & 0.03 \\
\hline$\lambda_{i-1} / \lambda_{i}(i>3)$ & & & & 0.84 & 0.88 & 0.66 & 0.81 & 0.68 & 0.86 & 0.9 & 0.94 & 0.95 & 0.82 & 0.91 & 0.95 & 0.9 & 0.89 \\
\hline
\end{tabular}

Because there are seven power plants in total, the partition number $\mathrm{K}$ that we should take is no greater than 7, there is no a unified method to determine spectral clustering algorithm $\mathrm{K}$, but in principle, it is to determine the difference of eigenvalues $\lambda$. Thus, $\mathrm{K}$ is determined by combining the analysis of the value of $\lambda$. Among them all, the node1,2,5,6,8,15 and16 are directly connected to the power plant line. Each partition method represents each partition in capital letters, as following table:

Table 4-4 Spectral clustering node partition table

\begin{tabular}{|c|l|l|l|l|l|l|l|l|l|l|l|l|l|l|l|l|l|}
\hline $\begin{array}{l}\text { Node } \\
\text { number }\end{array}$ & 1 & 2 & 3 & 4 & 5 & 6 & 7 & 8 & 9 & 10 & 11 & 12 & 13 & 14 & 15 & 16 & 17 \\
\hline$K=2$ & $A$ & $A$ & $A$ & $A$ & $B$ & $B$ & $B$ & $B$ & $B$ & $B$ & $B$ & $B$ & $B$ & $A$ & $A$ & $A$ & $A$ \\
\hline$K=3$ & $A$ & $A$ & $A$ & $A$ & $B$ & $B$ & $B$ & $B$ & $B$ & $B$ & $B$ & $C$ & $C$ & $C$ & $C$ & $C$ & $C$ \\
\hline$K=4$ & $A$ & $A$ & $A$ & $B$ & $B$ & $C$ & $C$ & $C$ & $C$ & $C$ & $C$ & $D$ & $D$ & $D$ & $B$ & $A$ & $A$ \\
\hline$K=5$ & $A$ & $A$ & $A$ & $B$ & $B$ & $C$ & $C$ & $C$ & $C$ & $D$ & $D$ & $D$ & $B$ & $B$ & $B$ & $E$ & $E$ \\
\hline$K=6$ & $A$ & $A$ & $A$ & $B$ & $B$ & $C$ & $C$ & $C$ & $C$ & $D$ & $D$ & $E$ & $E$ & $E$ & $B$ & $F$ & $F$ \\
\hline$K=7$ & $A$ & $A$ & $A$ & $B$ & $B$ & $B$ & $C$ & $C$ & $C$ & $D$ & $D$ & $E$ & $E$ & $F$ & $F$ & $G$ & $G$ \\
\hline
\end{tabular}

Note: In the table, the letters are numbered consecutively from the beginning of A.

\section{Partition validation}

Analysing the sample data from April 2nd to 27 (in this period of time the data of all nodes is all in readiness), first normalized, and then analyse the different nodes' time sequence similarity. Calculating nodes in different partitions with the Euclidean distance, DTW distance and Correlation distance, Manhattan distance, Cosine distance and so on to calculate each sub region node's similarity ${ }^{[15]}$. Steps are as follows:

1) Take7488 data of 17 nodes for 26 days to form a data matrix $M$ with 17 rows and 7488 columns; 
2) Use MATLAB to calculate the Euclidean distance, the DTW distance, the correlation coefficient distance, the Manhattan distance and the cosine distance of the 17 data lines, to get the distance matrix $P_{1}, P_{2}, P_{3}, P_{4}, P_{5}$;

3) Order each row of the distance matrix P1,P2,P3,P4,P5 respectively to obtain the corresponding distance of each node in the ascending order, getting the permutation matrix $Q_{1}, Q_{2}, Q_{3}, Q_{4}, Q_{5}$

4) As to the permutation matrix $Q_{1}, Q_{2}, Q_{3}, Q_{4}, Q_{5}$, combine the partition condition of the table 4-4, fill in the Table 5-1 to 5-4;

5) Define the concept of division degree, the degree of distinction means the differentiate degree of correlation between a partition node and the region and the nodes outside the region, to each partition, the formula is as follows:

$$
P=\frac{n u m}{n *(n-1)}
$$

The number of nodes in the zone that belongs to the partition is the total number of nodes in the partition. The following table is divided into two divisions of spectral clustering:

Table 5-1 spectral clustering two partition tables

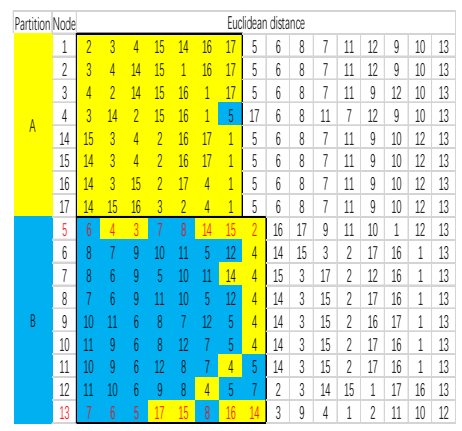

a. Euclidean distance

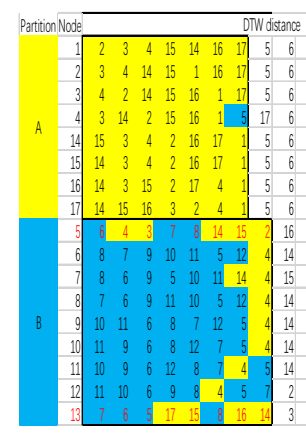

b.DTW distance

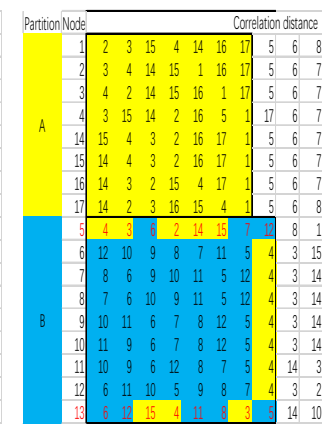

c. Correlation distance

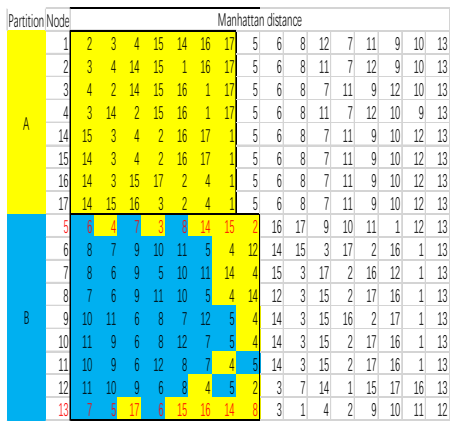

d. Manhattan distance

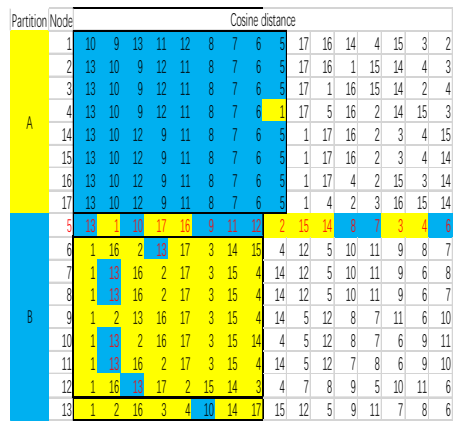

e. Cosine distance

Table 5-1 in various types of distance analysis (A) in the calculation of the number of partitions, and so on:

A. Euclidean distance: $=55 / 56=98.2 \% ;=55 / 72=76.4 \%$. Such as removing the influence area of 5, ranking No. 13 zigzag, node, $=48 / 56=85.7 \%$. If the impact of node 4 is not included (as a public node), then $=55 / 56=98.2 \%$.

B.DTW distance: because the time series data is consistent, the Euclidean distance is equivalent to the DTW distance theoretically, and the result is proved by the data in the table.

C. correlation coefficient distance: $=56 / 56=100.0 \%,=57 / 72=79.17 \%$. Remove 5 , node number 13 , then $=49 / 56=87.5 \%$. If the node number 4 is removed, the $=56 / 56=100.0 \%$.

D. Manhattan distance: $=56 / 56=100.0 \%,=51 / 72=70.83 \%$. Remove 5 , node number 13 , then $=45 / 56=80.4 \%$. Remove node 4 , then $=52 / 56=92.6 \%$. 
E. angle cosine distance: $=71 / 72=98.6 \%,=60 / 72=83.3 \%$. Remove 5 , node number 13 , then $=50 / 56=89.3 \%$, remove the number of nodes, then $=56 / 56=100.0 \%$.

To sum up, the conclusions are as follow:

1) In the case of no correction, the degree of differentiation is more than $70 \%$.

2) In the case where deviation exist, the boundary nodes are the main influencing factors, and these points are defined as key nodes, when the boundary nodes are more like the internet, there are strong associations with all partitions, which is called the vortex effect, such as node 4 and 5, and when the boundary nodes are connected by a single connection, the association is weak, which is called the island effect, such as No. 13 node. When the two cases are corrected, the difference is more than $80 \%$.

3) When the key nodes in 2) is seen as an individual part, the degree of differentiation can be up to more than $90 \%$.

\section{Conclusions}

Comprehensive analysis of the above discussion: The partition scheme has been determined to be two partitions, partition A includes: Node1,2,3,4,14,15,16 and 17; partition B contains: node $5,6,7,8,9,10,11,12$ and13. Node 4,5 with whiling effect are set up as special boundary zones, and the 13 node with island effect is set up as an autonomous node.

The four most important nodes, node 6 , node 12 , node 4 and node 14 discussed in section 3.5 are in each of the two partitions 4 and 14. In addition to the 4 nodes as the boundary node, the other 3 nodes are divided into internal nodes for easy controlling, and the number of nodes is located in the special boundary area, connected by a double route, the following programs can be referred:

1) When the 1,2,3,15,16,17 node occur big voltage fluctuations, first pay close attention to the change of the voltage in the partition $\mathrm{A}$, and pay attention to the voltage change in the special boundary area at the same time.

2) When the voltage of the node $7,8,9,10,11$ greatly fluctuate, the voltage change in the region $B$ should be paid close attention to, and the voltage variation in the special boundary region should be paid attention to.

3) When the voltage of the node 5 fluctuates greatly, it is necessary to pay attention to the change of the voltage in the region A and B, especially the voltage change near the special boundary.

4) When the voltage of the 13 node fluctuates greatly, it can be optimally and quickly controlled.

5) When the node 4,6,12and 14 have a large fluctuation, because of the high importance of these four nodes, it is necessary to consider the cross linkage control to ensure the voltage stability of these nodes.

\section{References}

[1] Li Gang et al. An anti overload control strategy based on graph partitioning and improved BFS algorithm to search for safety constraints [J]. proceedings of the Electrotechnical Society, 2012, 27 (219-229.).

[2] Xue Yusheng, Lai Ning. Fusion energy thinking and big data thinking big data (a) and the power of big data [J]. power system automation, 2016, 40 (1): 1-8.

[3] Yang Xuying, Zhou Ming. Analysis and modeling of demand response mechanism in smart grid [J]. power system technology, 2016, 40 (): 220-226. (in Chinese)

[4] Godsil, Chris. Algebraic graph theory. Vol. 207. Springer Science \& Business Media, 2013.

[5] pan Quan, Wang Zengfu, Liang Yan, et al. Basic methods and progress of information fusion theory (II) [J]. control theory and applications, 2012, 29 (): 599-615.

[6] Deo, Narsingh. Graph with to and computer Courier Dover Publications, 2016, applications, science. 
[7]Su Muya, Research on data mining of financial time series based on spectral clustering . Beijing: China Economic Publishing House, 2013

[8] he yangzan, temperature Zengyin. Power system analysis (upper and lower). Beijing: Huazhong University of Science and Technology press, 2002

[9] YANG Jian, TANG Fei, LIAO Qingfen, et al. Based on semi supervised spectral clustering for optimal active cross section search [J]. power system technology, 2015, 39 (): 242-249. (in Chinese)

[10] Zheng Yang Fan. Spectral clustering algorithm research and Realization of [D]. based on graph 2012 South China University of Technology.

[11] Wang Jing, Zhang Jinsuo. Comprehensive evaluation of several methods of determining weight vector comparison [J]. Journal of Hebei University of Technology, 2001, 30 (2): 52-57.

[12] Tan Yudong. Vulnerability assessment method for complex power system [D]. Hunan University, 2013

[13] Huang Faliang, Xiao industrial. The Web community spectral clustering comparison of [J]. Mini micro systems, 2011, 32 (4): 584-587.

[14] Lu Zong Zong. Review of microgrid research [J]. Automation of power systems, 2007, 31 (): 100-107.

[15] van Tu Yiming, Xionggang. A network node security domain division algorithm [J]. Journal of sensor technology based on spectral clustering analysis, 2014 (5): 675-679. 